\title{
Algorithms for classification of signals derived from Human Brain
}

\author{
Georgi P. Dimitrov \\ University of Library Studies and \\ Information Technologies \\ Sofia, Bulgaria \\ geo.p.dimitrov@gmail.com \\ Galina Panayotova \\ University of Library Studies and \\ Information Technologies, \\ Sofia, Bulgaria, \\ panayotovag@gmail.com \\ Boyan Jekov \\ University of Library Studies and \\ Information Technologies \\ Sofia, Bulgaria \\ b.jekov@unibit.bg
}

\author{
Pavel Petrov, \\ University of Economics \\ Varna, Bulgaria \\ petrov@ue-varna.bg \\ Iva Kostadinova \\ University of Library Studies and \\ Information Technologies \\ Sofia, Bulgaria \\ i.kostadinova@unibit.bg \\ Snejana Petrova \\ University of Library Studies and \\ Information Technologies \\ Sofia, Bulgaria \\ snejanapetrova_1977@abv.bg
}

\author{
Olexiy S. Bychkov \\ Taras Shevchenko National University \\ of Kyiv \\ Kyiv Ukraina \\ bos.knu@gmail.com \\ Vasyl Martsenyuk \\ vmartsenyuk@ath.bielsko.pl \\ Univerciti of Bielsko Biala \\ Poland \\ Aleksandar Parvanov \\ University of Library Studies and \\ Information Technologies \\ Sofia, Bulgaria \\ alexanderangelov@protonmail.com
}

Received: April 9, 2021. Received: September 6, 2021. Accepted: September 18, 2021. Published: September 20, 2021.

\begin{abstract}
Comparison of the Accuracy of different off-line methods for classification Electroencephalograph (EEG) signals, obtained from Brain-Computer Interface (BCI) devices are investigated in this paper.
\end{abstract}

BCI is a technology that allows people to interact directly or indirectly with their environment only by using brain activity. But, the method of signal acquisition is non-invasive, resulting in significant data loss. In addition, the received signals do not contain only useful information. All this requires careful selection of the method for the classification of the received signals.

The main purpose of this paper is to provide a fair and extensive comparison of some commonly employed classification methods under the same conditions so that the assessment of different classifiers will be more convictive. In this study, we investigated the accuracy of the classification of the received signals with classifiers based on AdaBoost (AB), Decision Tree (DT), k-Nearest Neighbor (kNN), Gaussian SVM, Linear SVM, Polynomial SVM, Random Forest (RF), Random Forest Regression ( RFR ). We used only basic parameters in the classification, and we did not apply fine optimization of the classification results.

The obtained results show suitable algorithms for the classification of EEG signals. This would help young researchers to achieve interesting results in this field faster.

Keywords- Machine Learning, Brain Wave, Artificial Intelligence, Mathematical models of objects and processes, Computer Science, Robotics

\section{INTRODUCTION}

Brain Computer Interface (BCI) systems create a communication bridge between the human brain and the outside world, eliminating the need for typical methods of delivering information. They control the sending of messages by the human brain and decode the transmitted signals.
Brain Computer Interface (BCI) enables users to communicate with multiple devices by detecting and processing EEG signals emitted by the human brain. The BCI system records brain waves and sends them to the computer system to perform the intended task. In this way, transmitted waves are used to identify the expression of an idea or the management of an object.

The use of BCI can enable different groups of users to control devices from our environment without using e.g. your hands. We just need to think about something, e.g. "Turn on the lights." This is enough to execute a set of commands and does not require muscular intervention [9], [10], [3]. For people with disabilities, using BCI as Assistant Robots can provide additional opportunities to make their daily lives easier. In the field of IT technologies, BCI can provide additional opportunities for the protection of information systems.

In order to implement a BCI system, various patterns of brain activity must be created that can be identified by the system and converted into commands. This means that the signals coming from the BCI must be processed and recognized correctly by the system.

Currently, off-line classification algorithms are most commonly used for this purpose. The design of the classification model involves the selection of one or more classification algorithms. However, in order for the assessment of classification to be sufficiently accurate, an appropriate algorithm must be selected, depending on the objective set for the researcher. The construction of a Real-Time Information system requires, in addition to the accuracy of signal recognition, a sufficiently high speed of signal processing, while for research purposes, high accuracy and reliability of the results are required.

In our study, we focus mainly on assessing the accuracy of the various algorithms. Usually, in different researches and articles, the work of one algorithm is shown, which gives a certain result, but we decided to compare the results of the work of the most 
frequently used algorithms, setting standard parameters under different conditions.

In this case, we investigated the accuracy of the classification of the received signals with classifiers based on AdaBoost (AB), Decision Tree (DT), k-Nearest Neighbor (kNN), Gaussian SVM, Linear SVM, Polynomial SVM, Random Forest (RF), Random Forest Regression ( RFR ).

\section{DESCRIPTION OF THE RESEARCH}

\section{A. Base description}

Various classical algorithms, such as BCI, are used to classify signals derived from BCI. However, only a few studies compare the effectiveness of these classifiers with different sample sizes for the same data set. In this study, we reviewed and compared the results obtained using $\mathrm{AB}, \mathrm{DT}$, kNN, Gaussian SVM, Linear SVM, Polynomial SVM, RF, and RFR classifiers to evaluate the accuracy in classifying BCI signals. All studies were performed with the same data set and each classifier was filled with 5 different types of parameters to find the optimal settings for each classifier.

In addition, in our study during the training process of the participant we use additional visual stimulation, which according to the results of our preliminary studies significantly increases the accuracy in classifying the data sent to the algorithm for analysis. This is due to the additional visual stimulus for concentration of the participants in the study..

The commands we researched here are the standard "Left" and "Right". The emitted brain waves were received by EMotiv Epoc 14+ device.

We used drivers CyKIT 3.0 for Python 3.x (github.com/hugofloresgarcia/CyKit) [7], [9]. The received signals were recorded in CSV format using OpenVibe platform (Inria Rennes, INSERM and Orange Labs in collaboration with AFM, CNRS, Gipsa-lab and CEA List). The process of classifier training and the following-after tests were performed in offline mode $[4,5,9]$. The data is processed in Python 3.9 - Spyder version 5.0.

In our research we focused only on Beta waves, between 12 and 30 hertz.

The standard experiment includes a visualization of an Arrow and a respective Written Command. In our case we had arrows and written commands for "Left" and "Right". The main point of interest in our experiment is connected to the respective reasoning of the participant when a particular sign and command appear on the screen: whether his main idea in this moment is the word "Left" or the word "Right'; whether the arrows for "Left" and "Right", or the phrases "Turn Left" and "Turn Right" $[12,16,17,18]$. It is significant that at different instances (moments) our brain can associate one and the same picture with different images and ideas having a common reference. It is for this reason that we also use additional visual stimulation during the experiment $[10,11]$, i.e. a simultaneous display on the screen of a respective command ( a real-time playing game - in our case - motorboat control).

The research includes analysis of raw data obtained from 16 physically and mentally healthy participants, without preexisting neurological disorders and previous experience with using Brain-Computer Interface (BCI) devices. The participants are of different age groups - between 21 and 54.

\section{B. Collected data}

Each experiment lasted 550 - $610 \mathrm{sec}$., or $\sim 10 \mathrm{~min}$.

There were $20 \mathrm{~min}$ intervals between the different stages in order to relax the participant. During the experiment, the respective images with written commands "left" and "right" were shown 20 times each. Each series consisted of a 3second display of the respective image (epoch) and additional visual and audio signals. At the beginning of the series, a 1-second beep was sounded to alert the participant. Each test series lasted 26 seconds [12]. This included 3 seconds to display the appropriate command and 20 seconds to perform synchronization actions. Because the experiment involved motor imagery, it was mainly focused on beta waves $(12-30 \mathrm{~Hz})$.

The data used in this experiment is collected from each participant using fourteen electrodes AF3, F7, F3, F5, T7, P7, O1, O2, P8, T8, FC6, F4, F8 and AF4 at locations of the motor cortex. The obtained raw data is shown in Table 1 .

TABLE I. RAW DATA

\begin{tabular}{|c|c|c|c|c|c|c|c|}
\hline Time:128Hz & Epoch & AF3 & F7 & & AF4 & $\begin{array}{l}\text { Event } \\
\text { Id } \\
\end{array}$ & $\begin{array}{l}\text { Event } \\
\text { Date } \\
\end{array}$ \\
\hline 0 & 0 & 4114.103 & 4110 & $\ldots$ & 4117.436 & & \\
\hline 0.0078125 & 0 & 4113.974 & 4107.692 & $\ldots$ & 4115.769 & 32769 & 0.008967 \\
\hline 0.015625 & 0 & 4107.82 & 4105.897 & & 4108.718 & & \\
\hline$\ldots \ldots$ & $\ldots$ & $\ldots$ & $\ldots$ & $\ldots$ & $\ldots$ & $\ldots$ & $\ldots$ \\
\hline 571.046875 & 0 & 4118.333 & 4111.41 & & 4120.128 & 33026 & 0.008967 \\
\hline
\end{tabular}

Dataset size is shown in Table 2 and parameter list for all algorithms in Table 3

TABLE II. DATASET SIZE

\begin{tabular}{|l|r|}
\hline & Row counts \\
\hline Number of data & $\sim 73000$ \\
\hline Of these for the commands left and right & 13800 \\
\hline Only for left / right & 6900 \\
\hline $\mathbf{2 0 \%}$ test sample & 1380 \\
\hline $\mathbf{3 0 \%}$ test sample & 2710 \\
\hline $\mathbf{4 0 \%}$ test sample & 2760 \\
\hline
\end{tabular}

TABLE III. PARAMETER LIST

\begin{tabular}{|c|c|c|}
\hline Classifier & Base parameters & $\begin{array}{r}\text { Additional } \\
\text { parameters }\end{array}$ \\
\hline AdaBoost Classifier & $\begin{array}{l}\text { n_neighbors }=1,20,50,70 \text {, } \\
100\end{array}$ & none \\
\hline DecisionTree Classifier & $\begin{array}{l}\text { n_neighbors }=1,20,50,70, \\
100\end{array}$ & none \\
\hline K-Neighbors Classifier & n_neighbors $=1,3,5,7,9$ & none \\
\hline $\begin{array}{r}\text { Gaussian SVM } \\
\text { Classifier }\end{array}$ & $\max _{100}$ dept $=1,20,50,70$ & $\mathrm{C}=10$ \\
\hline Linear SVM Classifier & $\begin{array}{r}\text { max_iter }=1000,3000,5000 \\
50000,10000\end{array}$ & $\mathrm{C}=10$, gamma='auto' \\
\hline 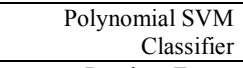 & degree $=2,4,6,8,10$ & $\begin{array}{r}\mathrm{C}=10, \text { max_iter } \\
5000, \text { gamma }=\text { 'auto' }\end{array}$ \\
\hline $\begin{array}{r}\text { Random Forest } \\
\text { Classifier }\end{array}$ & $\max \_$depth $=5,10,15,25,50$ & n_estimators $=10$ \\
\hline $\begin{array}{r}\text { Random Forest } \\
\text { Regressor Classifier }\end{array}$ & $\max \_d e p t=10,20,30,40,50$ & $\mathrm{n}$ estimators $=10$ \\
\hline
\end{tabular}




\section{Basic scheme}

The preliminary evaluation steps shown in Figure 1 are applied in order to increase the probability of accurate classification the received signals (movement, desire for something, etc.) from different channels (AF3, AF4, F3, F4, FC5, FC6, F7, O2 ...), responsible for various types of brain activity. The stages of data processing and classification algorithms are shown in Fig. 1

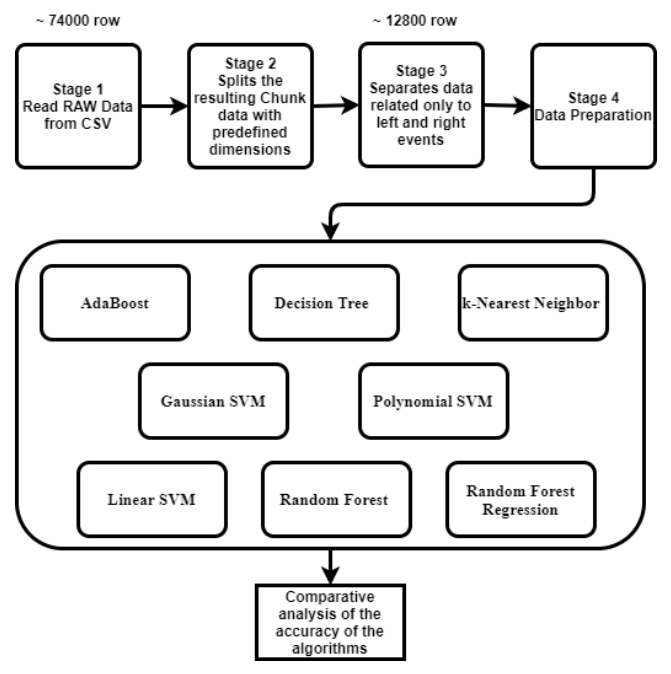

Fig. 1 Data processing and Classification algorithms

\section{Background theory}

AdaBoost algorithm (AB), short for Adaptive Boosting, is a Boosting technique that is used as an Ensemble Method in Machine Learning. It is called Adaptive Boosting as the weights are re-assigned to each instance, with higher weights to incorrectly classified instances. Boosting is used to reduce bias as well as the variance for supervised learning. It works on the principle where learners are grown sequentially. Except for the first, each subsequent learner is grown from previously grown learners. In simple words, weak learners are converted into strong ones.

Decision Trees (DTree) are a non-parametric supervised learning method used for classification and regression. The goal is to create a model that predicts the value of a target variable by learning simple decision rules inferred from the data features. A tree can be seen as a piecewise constant approximation[14].

$\mathrm{K}$ nearest neighbors $(\mathrm{KNN})$ : Instance based learning or lazy learning which trains the classifier function locally by majority note of its neighboring data points. Linear NN Search algorithm is used for search algorithm [14,25,28, 29].

A support vector machine (SVM) is a type of supervised machine learning classification algorithm that constructs one or more hyper planes to be can be used for classification[14]..

Gaussian RBF(Radial Basis Function) is Kernel method used in SVM models. RBF kernel is a function whose value depends on the distance from the origin or from some point.

Linear Kernel can be used as normal dot product any two given observations. The product between two vectors is the sum of the multiplication of each pair of input values[14].
Polynomial Kernel is a more generalized form of the linear kernel. The polynomial kernel can distinguish curved or nonlinear input space[14].

Random Forest (R. Forest) algorithm constructs a forest of random trees [38] with locations of attributes chosen at random. It uses an ensemble of unpruned decision trees by a bootstrap sample using training data. There is no restriction on the depth of the tree;

Random Forest Regression is a supervised learning algorithm that uses ensemble learning method for regression. Ensemble learning method is a technique that combines predictions from multiple machine learning algorithms to make a more accurate prediction than a single model[14].

The execution time of each algorithm was obtained from the CPU by calling the python function "time" before each start of the classification and after its execution.

To evaluate the result we use the indicator f-score.

The f-score showing weighted average of the precision and recall, where an f-score reaches its best value at 1 and worst score at 0 . The relative contribution of precision and recall to the f-score are equal. The formula for the f-score is:

$\mathrm{F} 1=2 *($ precision $*$ recall $) /($ precision + recall $)[14]$

\section{RESULT OF THE EXPERIMENT}

As mentioned above, as a result of the study, 4 data sets were taken from each participant, as a result, a total of $16 * 4$ $=64$ data sets were processed.

Each data set is processed sequentially with each of the algorithms - AdaBoost (AB), Decision Tree (DT), k-Nearest Neighbor (kNN), Gaussian SVM, Linear SVM, Polynomial SVM, Random Forest (RF), Random Forest Regression ( RFR ).

The result of Average F1-Score accuracy for dataset with $20 \%$ test size of data is shows on Table 4 .

TABLE IV. F1-SCORE - TEST SIZE - 20\%

\begin{tabular}{|l|c|c|c|c|c|}
\hline Algoritm_Type & Value 1 & Value 2 & Value 3 & Value 4 & Value 5 \\
\hline AB & $35.22 \%$ & $64.10 \%$ & $67.41 \%$ & $68.26 \%$ & $68.62 \%$ \\
\hline Dtree & $36.29 \%$ & $78.17 \%$ & $78.10 \%$ & $78.28 \%$ & $78.25 \%$ \\
\hline kNN & $\mathbf{9 0 . 1 6 \%}$ & $\mathbf{8 8 . 5 3 \%}$ & $\mathbf{8 7 . 8 8 \%}$ & $\mathbf{8 7 . 0 6 \%}$ & $\mathbf{8 6 . 6 2 \%}$ \\
\hline G SVM & $69.58 \%$ & $\mathbf{8 5 . 4 7 \%}$ & $\mathbf{8 8 . 2 1 \%}$ & $\mathbf{8 9 . 2 5 \%}$ & $\mathbf{8 9 . 7 9 \%}$ \\
\hline L SVM & $66.80 \%$ & $67.33 \%$ & $65.65 \%$ & $63.58 \%$ & $61.85 \%$ \\
\hline P SVM & $62.81 \%$ & $56.38 \%$ & $55.27 \%$ & $60.62 \%$ & $59.17 \%$ \\
\hline RF & $64.23 \%$ & $72.83 \%$ & $79.03 \%$ & $\mathbf{8 2 . 5 6 \%}$ & $\mathbf{8 1 . 4 8 \%}$ \\
\hline RFR & $16.79 \%$ & $36.97 \%$ & $42.21 \%$ & $41.58 \%$ & $42.42 \%$ \\
\hline
\end{tabular}

In the table, the results in bold with more than $80 \% \mathrm{~F} 1$ Score are indicated, and the colors in yellow - with over $90 \%$ F1 Score. In this case (test size - 20\%) only kNN and Gaussian SVM are suited for classification if we want classification accuracy over $85 \%$. For KNN the accuracy of the assessment is over $85 \%$ regardless of the number of $n$ neighbors, for G SVM the highest result is achieved at max_dept $=20,50,70$ and 100 . It should be borne in mind that $\overline{i t}$ is not good to use $n \_$neighbors $=1$ for classification[]. 
A result of over $80 \%$ was also obtained for Random Forrest algorithm whit max_dept over 15 .

The diagram in Figure 2 shows the summarized results for the accuracy of the classified signals for all algorithms at train size 80 and test size $20 \%$ in datasets.

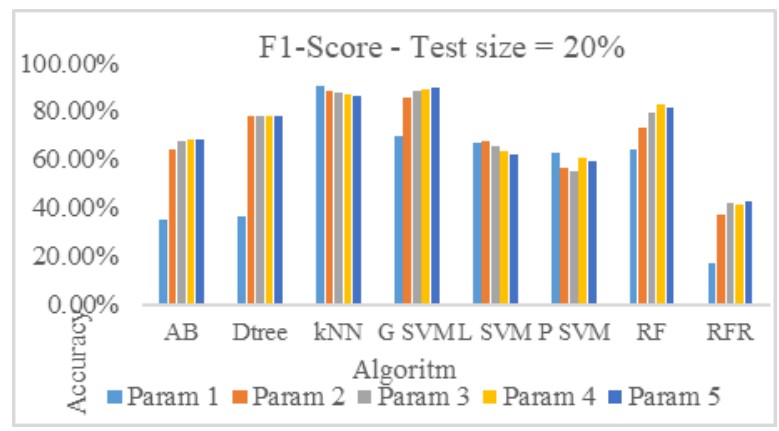

Fig. 2 Summarize result - test size $=20 \%$

The result of Average F1-Score accuracy for dataset with $30 \%$ test size of data is shows on Table $\mathrm{V}$.

TABLE V. AVG ACCURACI - F1-SCORE - TEST SIZE - 30\%

\begin{tabular}{|l|r|r|r|r|r|}
\hline Algoritm_Type & Param 1 & Param 2 & Param 3 & Param 4 & Param 5 \\
\hline AB & $35.58 \%$ & $62.88 \%$ & $67.95 \%$ & $68.39 \%$ & $68.92 \%$ \\
\hline Dtree & $11.08 \%$ & $77.06 \%$ & $77.17 \%$ & $77.06 \%$ & $77.29 \%$ \\
\hline kNN & $\mathbf{8 8 . 9 0 \%}$ & $\mathbf{8 7 . 8 3 \%}$ & $\mathbf{8 6 . 8 5 \%}$ & $\mathbf{8 6 . 3 3 \%}$ & $\mathbf{8 5 . 8 3 \%}$ \\
\hline G SVM & $69.19 \%$ & $84.91 \%$ & $\mathbf{8 8 . 0 3 \%}$ & $\mathbf{8 9 . 1 0 \%}$ & $\mathbf{9 0 . 1 1 \%}$ \\
\hline L SVM & $67.29 \%$ & $67.29 \%$ & $64.24 \%$ & $60.64 \%$ & $61.08 \%$ \\
\hline P SVM & $58.93 \%$ & $53.39 \%$ & $41.88 \%$ & $33.87 \%$ & $33.82 \%$ \\
\hline RF & $64.37 \%$ & $72.46 \%$ & $79.43 \%$ & $\mathbf{8 2 . 4 6 \%}$ & $\mathbf{8 1 . 5 3 \%}$ \\
\hline RFR & $17.16 \%$ & $38.44 \%$ & $41.46 \%$ & $42.09 \%$ & $40.24 \%$ \\
\hline
\end{tabular}

In this case (test size - 30\%) only $\mathrm{kNN}$ and Gaussian SVM are suited for classification if we want classification accuracy over $85 \%$. For KNN the accuracy of the assessment is over $85 \%$ regardless of the number of $n$ neighbors, for G SVM the highest result is achieved at max_dept $=50,70$ and 100 .

The diagram in Figure 3 shows the summarized results for the accuracy of the classified signals for all algorithms at train size 70 and test size $30 \%$ in datasets.

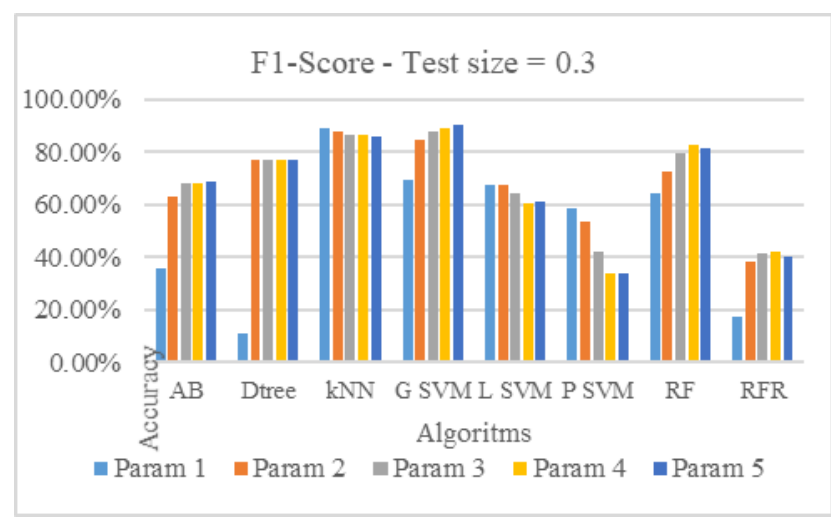

Fig. 3 Summarize result - test size $=30 \%$

In this case in Table VI - (test size - 40\%) again $\mathrm{kNN}$ and Gaussian SVM showing accuracy over $85 \%$. For KNN the accuracy of the assessment is over $85 \%$ regardless of the number of n-neighbors, for G SVM again the highest result is achieved at max_dept $=20,50,70$ and 100 .

TABLE VI. AVG ACCURACI - F1-SCORE - TEST SIZE - 40\%

\begin{tabular}{|l|r|r|r|r|r|}
\hline Algoritm_Type & Param 1 & Param 2 & Param 3 & Param 4 & Param 5 \\
\hline AB & $11.63 \%$ & $63.11 \%$ & $68.64 \%$ & $69.00 \%$ & $69.57 \%$ \\
\hline Dtree & $34.35 \%$ & $75.49 \%$ & $76.53 \%$ & $76.61 \%$ & $76.29 \%$ \\
\hline kNN & $\mathbf{8 8 . 3 9 \%}$ & $\mathbf{8 7 . 2 4 \%}$ & $\mathbf{8 6 . 5 5 \%}$ & $\mathbf{8 5 . 7 7 \%}$ & $\mathbf{8 4 . 9 7 \%}$ \\
\hline G SVM & $69.34 \%$ & $\mathbf{8 4 . 2 7 \%}$ & $\mathbf{8 7 . 2 4 \%}$ & $\mathbf{8 7 . 8 3 \%}$ & $\mathbf{8 8 . 5 8 \%}$ \\
\hline L SVM & $67.23 \%$ & $67.01 \%$ & $61.70 \%$ & $61.05 \%$ & $61.10 \%$ \\
\hline P SVM & $60.06 \%$ & $52.26 \%$ & $40.73 \%$ & $33.86 \%$ & $33.86 \%$ \\
\hline RF & $62.65 \%$ & $71.51 \%$ & $79.07 \%$ & $\mathbf{8 1 . 7 7 \%}$ & $\mathbf{8 1 . 3 0 \%}$ \\
\hline RFR & $15.14 \%$ & $36.28 \%$ & $41.66 \%$ & $41.26 \%$ & $41.69 \%$ \\
\hline
\end{tabular}

Below are the summarized results for the two algorithms that showed the highest results - $\mathrm{kNN}$ - Table VII and Gausian SVM - Table VIII

TABLE VII. KNN - F1- SCORE

\begin{tabular}{|c|r|r|r|r|r|}
\hline Test size & Param 1 & Param 2 & Param 3 & Param 4 & Param 5 \\
\hline $\mathbf{0 . 2}$ & $90.16 \%$ & $88.53 \%$ & $87.88 \%$ & $87.06 \%$ & 0.86616281 \\
\hline $\mathbf{0 . 3}$ & $88.90 \%$ & $87.83 \%$ & $86.85 \%$ & $86.33 \%$ & 0.858326895 \\
\hline $\mathbf{0 . 4}$ & $88.39 \%$ & $87.24 \%$ & $86.55 \%$ & $85.77 \%$ & 0.849727973 \\
\hline
\end{tabular}

TABLE VIII. GAUSSIAN SVM - F1- SCORE

\begin{tabular}{|c|r|r|r|r|r|}
\hline Test size & Param 1 & Param 2 & Param 3 & Param 4 & \multicolumn{1}{c|}{ Param 5 } \\
\hline $\mathbf{0 . 2}$ & $69.58 \%$ & $85.47 \%$ & $88.21 \%$ & $89.25 \%$ & $89.79 \%$ \\
\hline $\mathbf{0 . 3}$ & $69.19 \%$ & $84.91 \%$ & $88.03 \%$ & $89.10 \%$ & $90.11 \%$ \\
\hline $\mathbf{0 . 4}$ & $69.34 \%$ & $84.27 \%$ & $87.24 \%$ & $87.83 \%$ & $88.58 \%$ \\
\hline
\end{tabular}

From the obtained results it is clear that the optimal test sample (train set) in $\%$ is $30 \%$ or $40 \%$, and the number of neighbors -5 form kNN. For Gaussian SVN we need set max_dept parameter over 20 .

Overall performance measure of classification algorithms on EEG datasets is showing on Table IX

TABLE IX. OVERALL PERFORMANCE MEASURE

\begin{tabular}{|l|r|r|r|r|r|}
\hline Algoritm_Type & Param 1 & Param 2 & Param 3 & Param 4 & Param 5 \\
\hline AB & $35.58 \%$ & $64.10 \%$ & $68.64 \%$ & $\mathbf{6 9 . 0 0 \%}$ & $69.57 \%$ \\
\hline Dtree & $36.29 \%$ & $78.17 \%$ & $78.10 \%$ & $\mathbf{7 8 . 2 8 \%}$ & $78.25 \%$ \\
\hline kNN & $\mathbf{9 0 . 1 6 \%}$ & $88.53 \%$ & $87.88 \%$ & $87.06 \%$ & $86.62 \%$ \\
\hline G SVM & $69.58 \%$ & $85.47 \%$ & $88.21 \%$ & $89.25 \%$ & $90.11 \%$ \\
\hline L SVM & $67.29 \%$ & $67.33 \%$ & $65.65 \%$ & $63.58 \%$ & $\mathbf{6 1 . 8 5 \%}$ \\
\hline P SVM & $\mathbf{6 2 . 8 1 \%}$ & $56.38 \%$ & $55.27 \%$ & $60.62 \%$ & $59.17 \%$ \\
\hline RF & $64.37 \%$ & $72.83 \%$ & $79.43 \%$ & $\mathbf{8 2 . 5 6 \%}$ & $81.53 \%$ \\
\hline RFR & $17.16 \%$ & $38.44 \%$ & $42.21 \%$ & $42.09 \%$ & $\mathbf{4 2 . 4 2 \%}$ \\
\hline
\end{tabular}

and the graph of the obtained results is showing Fig. 6 


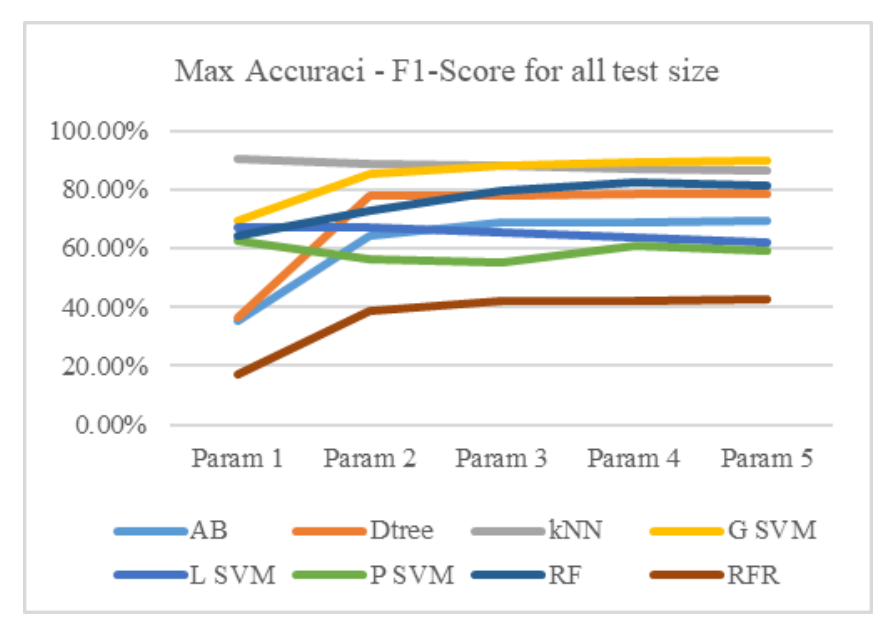

Fig. 6 Max F1-Score

In Table $\mathrm{X}$ is showing the best parameters and execution times for each of the algorithms.

TABLE X. MAX ACCURACI - F1-SCORE FOR ALL TEST SIZEL

\begin{tabular}{|l|l|r|}
\hline & \multicolumn{1}{|c|}{ Best parameters } & Time Execution \\
\hline AB & neighbors $=100$ & 0.126827478 \\
\hline Dtree & neighbors $=100$ & 0.018254995 \\
\hline kNN & $\begin{array}{l}\text { n_neighbors }=1,3,5,7,9 \text { - no matter, but } \\
\text { the best }-1\end{array}$ & 1.081873178 \\
\hline G SVM & max_dept $=$ all $>20$ & 7.504461765 \\
\hline LSVM & max_iter $=3000$ & 2.599415779 \\
\hline P SVM & max_iter $=2$ & 3.116208911 \\
\hline RF & max_depth $=25,50$ & 0.019266009 \\
\hline RFR & max_dept $=30$ & 0.017406821 \\
\hline
\end{tabular}

\section{CONCLUSION}

We studied 8 different types of classification algorithms, including AdaBoost (AB), Decision Tree (DT), k-Nearest Neighbor (kNN), Gaussian SVM, Linear SVM, Polynomial SVM, Random Forest (RF), Random Forest Regression ( RFR ) meta-analysis, and compared their results in classifying EEG signals using a different number of characteristics. We found that the k-Neighbor Neighbor and Gaussian SVM classification algorithms outperformed most classification algorithms, as the k- Nearest Neighbor algorithm is faster.

The obtained results show suitable algorithms for the classification of EEG signals. This will help young researchers to achieve interesting results in this area faster.

\section{ACKNOWLEDGMENT}

This work is supported by the research program PPNIP2021-09/12.14.2021 "Analysis and optimization of algorithms for classification of signals coming from Smart IoT devices" and National Science Program "Information and communication technologies for unified digital market in science".

\section{REFERENCES}

[1] Dr. Zhibin Tan, Dr. William H. Blanton, Miss Qianru Zhang, "Real-time EEG signal processing based on TI's
TMS320C6713 DSK", 120th ASEE Annual Conference@Exposition, Frankly, 23-26 Jone, 2013

[2] B Colombet, M Woodman, C G Bénar, J M Badier, "AnyWave: A cross-platform and modular software for visualizing and processing electrophysiological signals", HAL Id: hal-01323171, https://hal.archivesouvertes.fr/hal-01323171, Submitted on 30 May

[3] Elsawy, A.S., Eldawlatly, S.: P300-based Applications for Interacting with Smart Mobile Devices. In: 7th Annual International IEEE EMBS Conference on Neural Engineering. (2015) 166 - 169

[4] Fabien Lotte, Laurent Bougrain, Andrzej Cichocki, Maureen Clerc, Marco Congedo, et al.. A Review of Classification Algorithms for EEG-based BrainComputer Interfaces: A 10-year Update. Journal of Neural Engineering, IOP Publishing, 2018, 15 (3), pp.55. 10.1088/1741-2552/aab2f2. hal-01846433;

[5] Fabien Lotte, Marco Congedo, Anatole Lécuyer, Fabrice Lamarche, Bruno Arnaldi. A review of цlassification algorithms for EEG-based braincomputcr interfaces. Journal of Neural Engineering,IOP Publishing, 2007, 4, pp.24. inria-00134950

[6] Jasper, H.H.: The ten twenty electrode system of the international federation. Electroencephalography and clinical neurophysiology 10 (1958) 371 - 375

[7] Galina S. Panayotova, Georgi P. Dimitrov; Analysis of the BCI signals; 2018; 5th International Conference on Communications and Engineering (ICCNE2018), December 21-23, 2018, Paris, France.

[8] G. Dimitrov et al., "Increasing the Classification Accuracy of EEG based Brain-computer Interface Signals," 2020 10th International Conference on Advanced Computer Information Technologies (ACIT), Deggendorf, Germany, 2020, pp. 386-390, doi: 10.1109/ACIT49673.2020.9208944.

[9] Georgi P. Dimitrov, Galina Panayotova , Eugenia Kovatcheva, Pepa Petrova, Kristian V. Aleksiev, Inna Dimitrova, Pavel Petrov; Influence of the Length of the Epochs for the Correct Classification of Brain Waves in the OpenVibe Platform $2019 \quad$ International Academic Conference on Global Education, Teaching and Learning in Vienna 2019 (IAC-GETL 2019); ISBN 978-80-88203-14-8

[10] Georgi P. Dimitrov, Magdalena Garvanova, Eugenia Kovatcheva , Kristian V. Aleksiev, Inna Dimitrova; Identification of EEG Brain Waves obtained by Emotive device; 9th International Conference on Advanced Computer Information Technologies (ACIT) ; http://acit.tneu.edu.ua/;Electronic ISBN: 978-17281-0450-8; Print ISBN: 978-1-7281-0449-2; ISBN: 978-1-7281-0451-5; 10.1109/ACITT.2019.8779861;

[11] G. Panayotova, G.P. Dimitrov, "Modeling and data processing of information systems",Published in: Artificial Intelligence and Pattern Recognition (AIPR), International Conference on, Date of Conference: 19-21 Sept. 2016, Date Added to IEEE Xplore: 13 October 2016, ISBN: 978-1-4673-9187-0; CD-ROM ISBN: 9781-4673-9186-3, (PoD) ISBN: 978-1-4673-9188-7

[12] Georgi P. Dimitrov, Galina S. Panayotova, Eugenia Kovatcheva, Daniela Borissova, and Pavel Petrov, One approach for identification of Brain Signals for smart devices control , ,2018 2nd International Conference on 
Computer, Software and Modeling (ICCSM 2018) July 17-19, 2018, Nice, France, SW 2018 Vol.13(7): p. 407413 , ISSN: 1796-217X, doi: 10.17706/ jsw.13.7.407413, Journal of Software (JSW) - Volume 13 Number 7 (Jul. 2018)

[13] Georgi Dimitrov, Oleksiy Bychkov, Pavel Petrov, "One Approach for Analysis of Fuzzy Linear Hybrid Automata", Izvestia Journal of the Union of ScientistsVarna. Economic Sciences Series, t. 7, p. 234240,Union of Scientists-Varna, Economic Sciences Section, Print ISSN: 1314-7390, Online-ISSN: 26034085

[14] Katona, J., Peter, D., Ujbanyi, T., Kovari, A.: Control of incoming calls by a Windows Phone based Brain Computer Interface. 15th IEEE International Symposium on Computational Intelligence and Informatics (CINTI 2014) (2014) 121-125

[15] Kukreja, M., Johnston, S.A. \& Stafford, P. Comparative study of classification algorithms for immunosignaturing data. BMC Bioinformatics 13, 139 (2012). https://doi.org/10.1186/1471-2105-13-139

[16] Muhammad N. Fakhruzzamana, Edwin Riksakomara b, Hatma Suryotrisongko,"EEG Wave Identification in Human Brain with Emotiv EPOC for Motor Imagery", The Third Information Systems International Conference, Procedia Computer Science 72 ( 2015 ) $269-276$

[17] Petrov, P., Dimitrov, P., Petrova, S. Geohash-EAS - a Modified Geohash Geocoding System with Equal-Area Spaces. SGEM 2018 : 18 International Multidisciplinary Scientific Geoconference : Conference Proceedings, Sofia : STEF92 Technology Ltd., Vol.18 Informatics, Geoinformatics a. Remote Sensing, 2018, Iss. 2.2, 187 - 194.
[18] PfurtschellerG, NeuperC, FlotzingerD, PregenzerM. EEG-based discrimination between imagination of right and left hand movement. Electroencephalogr Clin Neurophysiol 1997;103:642-51. https://doi.org/10.1016/S0013-4694(97)00080-1.

[19] R.Bousseta, I.E.Ouakouak, M.Gharbi, F.Regragui, "EEG Based Brain Computer Interface for Controlling a Robot Arm Movement Through Thought", IRBM 39(2018)129-135

[20] Tabov, J., G. Panayotova. Chronological modeling of the West - European information about the medieval maps of the Ottoman world 16th -18 th Centuries. IJ Information Models \& Analyses, Vol. 3/ 2014, No. 3, 247-251. ISSN 1314-6416 (printed) ISSN 1314-6432 (Online) [www.foibg.com]

[21] TayebS, MahmoudiA, RegraguiF, HimmiMM. Efficient detection of P300 using Kernel PCA and support vector machine. In: 2014 second World conf complex syst. IEEE; 2014. p.17-22.

[22] Wang, Y.T., Wang, Y., Jung, T.P.: A cell-phone-based brain-computer interface for communication in daily life. Journal of neural engineering 8 (2011) 025 - 018

[23] Willian Dimitrov, Roumen Nikolov, and Boyan Jekov. STANDARDS AND INTEROPERABILITY WITHIN SMART CITY ECOSYSTEM. In ICERI2017 Proceedings. IATED, nov 2017

[24] V. Martnez-Cagigal et al. Schalk, G., McFarland, D.J., Hinterberger, T., Birbaumer, N., Wolpaw, J.R.: BCI2000: A general-purpose brain-computer interface (BCI) system. IEEE Transactions on Biomedical Engineering 51(6) (2004) 1034 - 1043

[25] https://www.javatpoint.com/k-nearest-neighboralgorithm-for-machine-learning

\section{Creative Commons Attribution License 4.0 (Attribution 4.0 International, CC BY 4.0)}

This article is published under the terms of the Creative Commons Attribution License 4.0 https://creativecommons.org/licenses/by/4.0/deed.en_US 